\title{
Signaling Dynamics of TSHR-Specific CAR-T Cells Revealed by FRET-Based Biosensors
}

\author{
Jing Zhou ${ }^{1,2}$, Jiangqing Chen ${ }^{3,4,5}$, Yanjie Huang ${ }^{6}$, Xiaofei Gao ${ }^{6}$, Chun Zhou ${ }^{7}$, \\ Xianhui Meng ${ }^{3,4,5 *}$ and Jie Sun ${ }^{3,4,5 *}$
}

${ }^{1}$ Department of Breast and Thyroid Surgery, Union Hospital, Tongji Medical College, Huazhong University of Science and Technology, Wuhan, China, ${ }^{2}$ Department of Breast and Thyroid Surgery, People's Hospital of Dongxihu District Wuhan City and Union Dongxihu Hospital, Huazhong University of Science and Technology, Wuhan, China, ${ }^{3}$ Liangzhu Laboratory, Zhejiang University Medical Center, Hangzhou, China, ${ }^{4}$ Department of Cell Biology, Bone Marrow Transplantation Center of the First Affiliated Hospital, Zhejiang University School of Medicine, Hangzhou, China, ${ }^{5}$ Institute of Hematology, Zhejiang University and Zhejiang Engineering Laboratory for Stem Cell and Immunotherapy, Hangzhou, China, ${ }^{6}$ Key Laboratory of Structural Biology of Zhejiang Province, School of Life Sciences, Westlake University, Hangzhou, China, ${ }^{7}$ School of Public Health, Sir Run Run Shaw Hospital, Zhejiang University School of Medicine, Hangzhou, China

OPEN ACCESS

Edited by: Yingxiao Wang, University of California, San Diego, United States

Reviewed by: Kazuhiro Aoki,

Graduate University for Advanced Studies (Sokendai), Japan Tian Chi,

Yale University, United States

${ }^{*}$ Correspondence: Xianhui Meng xianhm@zju.edu.cn Jie Sun sunj4@zju.edu.cn

Specialty section: This article was submitted to Cell Adhesion and Migration,

a section of the journal Frontiers in Cell and Developmental Biology

Received: 29 December 2021 Accepted: 31 January 2022 Published: 17 February 2022

Citation:

Zhou J, Chen J, Huang Y, Gao X, Zhou C, Meng $X$ and Sun J (2022) Signaling Dynamics of TSHR-Specific CAR-T Cells Revealed by FRETBased Biosensors. Front. Cell Dev. Biol. 10:845319. doi: 10.3389/fcell.2022.845319
Although most patients with thyroid cancers have good prognosis and long-term survival, some patients are refractory to traditional therapeutic approaches and face a high risk of mortality. CAR-T therapy provides an attractive strategy to treat these patients. Considering the limited expression in thyroid tissues, thyroid-stimulating hormone receptor (TSHR) has been considered as a promising candidate as CAR-T target. However, it is still a challenge to find the optimal CAR design for the treatment of thyroid cancers. Dynamic signaling cascade is initiated by CAR molecules during CAR-T cell activation. The development of FRET-based biosensors enables us to detect the signaling dynamics of key kinases during CAR-T cell activation with high spatiotemporal resolution. Here using the ZAP70 and ERK biosensors, we visualized the dynamics of ZAP70 and ERK activities in TSHR-specific CAR-T cells upon antigen stimulation. We first constructed several TSHR-targeting CARs for the treatment of advanced thyroid cancers. The TSHR CAR-T cells with CD28 or 4-1BB co-stimulatory signaling domains exhibited potent cytotoxicity in vitro. By FRET imaging, we observed rapid increase of ZAP70 and ERK activities in TSHR CAR-T cells upon target cell binding. Even though CD28based CAR-T cells had similar ZAP70 activation dynamics as 4-1BB-based CAR-T cells, they displayed slightly enhanced ERK activation, which may contribute to their faster anti-tumor kinetics in vivo. These results demonstrated the efficacy of TSHR CAR-T cells to treat advanced thyroid cancers. Our study indicated the potential of applying FRET biosensors to optimize the design of CAR for effective CAR-T therapy.

\section{Keywords: FRET, ERK, ZAP70, CAR-T, TSHR, thyroid cancer}

\section{INTRODUCTION}

Thyroid cancer is the most common endocrine cancer and accounts for 3\% of the new cancer cases worldwide in 2020 (Sung et al., 2021). Majority of thyroid cancers are differentiated thyroid cancers (DTC, 94\%), which mainly include papillary thyroid cancer (PTC, $80 \%$ of all cases), and follicular thyroid cancer (FTC, $15 \%$ of all cases). Although most patients with DTCs have excellent prognosis after radical surgery and radioactive iodine (RAI) treatment, a small fraction of them (about 20\%) 
may develop into advanced stage DTCs after tumor recurrence and metastasis, which may require reoperation. Large amount of fibrous and scar tissue in the surgical field will bring difficulties to the reoperation, which increases the incidence of permanent nerve injury and hypoparathyroidism, especially for patients who needs central lymph nodes dissection and/or cervical lymph nodes dissection again. It is reported that the incidence of permanent hypocalcemia and permanent vocal cord paralysis after re-dissection of cervical lymph nodes can be as high as 4.9 and $17.8 \%$ respectively, which may seriously affect the quality of patients' life. For such patients, exploring a new non-invasive treatment method is of great value. In addition, $5-10 \%$ of DTC cases with recurrence and metastasis are resistant to traditional therapy and suffer a high risk of mortality. It is reported that patients with refractory DTCs have less than $50 \%$ of 5 -years survival rate (Laha et al., 2020). For these patients, it is urgent to explore new treatment methods.

Chimeric antigen receptor $\mathrm{T}(\mathrm{CAR}-\mathrm{T})$ therapy represents a novel therapeutic approach and has achieved significant progress in treating hematological malignancies. The canonical CAR structure includes an $\mathrm{scFv}$ domain responsible for antigen recognition, a hinge and transmembrane domain, and a combined co-stimulatory and activation domain that initiates $\mathrm{T}$ cell activation. CAR molecules can reprogram $\mathrm{T}$ cell to recognize and eliminate tumor cells expressing specific antigens (van der Stegen et al., 2015). Although many challenges remain, experimental and clinical studies have shown some positive outcomes of CAR-T therapy for solid tumors (Newick et al., 2017). For advanced thyroid cancers, recent studies demonstrated that CAR-T therapy has potential therapeutic efficacy in treating anaplastic thyroid cancer (ATC) and medullary thyroid cancer (MTC) in preclinical models (Min et al., 2017; Bhoj et al., 2021).

Successful application of CAR-T therapy requires specific recognition of tumor cells. However, most solid tumors, including thyroid cancer, lack tumor-specific antigens that could be used to precisely distinguish tumor and normal tissue cells. Some tumor-associated antigens (TAA) have been used as CAR-T cell targets, while the risk of tumor relapse or severe on-target/off-tumor toxicity has to be evaluated carefully. As an alternative, lineage-specific antigens may be considered as targets of CAR-T cells in solid tumors as long as complete tissue eradiation is tolerant (Newick et al., 2017; Kosti et al., 2018). The thyroid-stimulating hormone receptor (TSHR) is a surface glycoprotein receptor whose expression is highly restricted to thyroid tissues. Evidence has shown continued high expression of TSHR in the majority of DTCs, including $90.8 \%$ in PTC, $89.2 \%$ in FTC, $78.2 \%$ in the cervical lymph node metastases, and $86.7 \%$ in the RAI-resistant metastases. These features suggest the potential of TSHR as CAR-T therapy targets for the treatment of advanced stage DTCs (Davies et al., 2002; Rowe et al., 2017).

Currently, the second-generation CARs that incorporate CD28 or $4-1 \mathrm{BB}$ signaling domains are the most prevalent CAR designs. Both CD28 and 4-1BB-based CAR-T cells can achieve complete tumor eradiation in clinical studies of refractory B cell malignancies. However, the different co-stimulatory domains have endowed CAR-T cells with distinct anti-tumor kinetics. CD28-based CAR-T cells often show rapid tumor eradiation with less persistence. In contrast, 4-1BB-based CAR-T cells often have less cytotoxic effect while persist longer in vivo (Feucht and Sadelain, 2020). Therefore, in the context of different solid tumors, the anti-tumor functions of CD28 and 4-1BB-based CARs may vary.

Nowadays, the development of genetically-encoded, FRETbased biosensors makes it possible to investigate kinetics of key signaling molecules in live cells with high spatiotemporal resolution. Recent studies have demonstrated that FRET-based biosensors can be used to monitor dynamics of some key molecules responsible for $\mathrm{T}$ cell activation (Xiang et al., 2011; Li et al., 2016; Wan et al., 2019). Particularly, CAR structures with modified $\mathrm{CD} 3 \zeta$ domains were shown to induce different Zetachain-associated protein kinase 70 (ZAP70) activation kinetics in CAR-T cells upon antigen stimulation (Liu et al., 2021).

In this study, we designed CAR-T cells targeting TSHR and evaluated their efficacy for the treatment of metastatic thyroid cancers both in vitro and in vivo. Using FRET-based biosensors, we further quantified and compared the dynamics of ZAP70 and Extracellular signal-regulated kinase (ERK) signals in CD28 and 4-1BB-based CAR-T cells upon antigen stimulation. As a member of the Syk family of thrysine kinases, ZAP70 plays a crucial role in regulating $\mathrm{T}$ cells activation. After antigen engagement, ZAP70 is recruited to the phosphorylated $\mathrm{CD} 3 \zeta$ subunits, where it is activated by LCK kinase and facilitates downstream signal amplification (Wang et al., 2010). Previous studies have suggested ZAP70 signal as a rate-limiting step in CAR-T cells activation. Insufficient activation of ZAP70 signal was thought to impair CAR-T cells functions (Gudipati et al., 2020). The ERK signal is considered as one of the main hubs to transduce proximal signals into nucleus during $\mathrm{T}$ cell activation. It is thought to exhibit a switch-like functions and the transient or persistent ERK dynamics could induce distinct gene expressions. The dynamics of ERK signal have been finely tuned by spatiotemporal networks to control cell fate decisions (Purvis and Lahav, 2013; Rohrs et al., 2020). Inside T cells, the ERK pathway is initiated by Rac-1 after the activation of Linker for activation of T cells (LAT), whose phosphorylation by ZAP70 makes it recruit multiple effector molecules for signal propagation. We found that TSHR binding elicits activation of ZAP70 and ERK in both CD28 and 4-1BB-based TSHR CAR-T cells. While two types of CAR-T cells had similar ZAP70 activity, different co-stimulatory signals resulted in distinctive ERK activation dynamics. This may be responsible for their different anti-tumor kinetics in mice.

\section{MATERIALS AND METHODS}

\section{Cell Lines}

293T cells, Jurkat T cells and K562 cells were obtained from ATCC. Thyroid cancer cell line $8505 \mathrm{c}$ cells was obtained from DSMZ (German Collection of Microorganisms and Cell Cultures). 293T cells and 8505c cells were cultured in high glucose DMEM medium with 10\% fetal bovine serum (FBS) 
and $1 \%$ penicillin/streptomycin $(\mathrm{P} / \mathrm{S})$. Jurkat T cells (clone E6-1) and $\mathrm{K} 562$ cells were cultured in IMDM medium with 10\% FBS and $1 \% \mathrm{P} / \mathrm{S}$. All cells were maintained in $5 \% \mathrm{CO}_{2}$ at $37^{\circ} \mathrm{C}$.

\section{Plasmids Construction}

All plasmids were constructed using ClonExpress II One Step Cloning Kit (Vazyme, Nanjing, China) following manufacturer's instruction. Retrovirus plasmids encoding TSHR-specific CARs were constructed by inserting the CAR constructs into SFG $\gamma$-retroviral vector. For detection of CAR expression, a sequence encoding green fluorescence protein (GFP) was linked in the C-terminus of CAR by a P2A peptide. Plasmids used for cell line transduction were constructed in lentiviral vectors. To prepare the plasmid co-expressing TSHR and luciferase, the sequences encoding TSHR and luciferase reporter were linked by a P2A peptide. The ZAP70 and ERK biosensors were reported by previous studies. The ZAP70 biosensor used the peptide (SREYACISGEL) as ZAP70 substrate (Liu et al., 2021). The ERK biosensor used the peptide (PDVPRTPVDKAKLSFQFPF) as ERK substrate (Komatsu et al., 2011). For co-expression of ZAP70 biosensor or ERK biosensor and CARs in Jurkat $\mathrm{T}$ cells, the CAR construct was introduced in the $\mathrm{C}$-terminus of biosensor sequence using a P2A peptide.

\section{T Cell Isolation and Transduction}

Human blood was obtained from healthy donors with written approval. PBMC was isolated using human lymphocyte isolation kit (Dakewe, Shenzhen, China). T cells were further purified using Pan $\mathrm{T}$ cell isolation kit (Miltenyi Biotec, Bergisch Gladbach, Germany) and cultured with X-VIVO 15 medium (Lonza) supplemented with $10 \%$ FBS, $1 \% \quad \mathrm{P} / \mathrm{S}, \quad 5 \mathrm{ng} / \mathrm{ml} \quad \mathrm{IL}-7$ (Novoprotein, Shanghai, China) and $5 \mathrm{ng} / \mathrm{ml}$ IL-15 (Novoprotein). Immediately after $\mathrm{T}$ cell isolation, they were stimulated with CD3/CD28 T cell Activator Dynabeads (Invitrogen, Carlsbad, United States) at a ratio of 1:1. T cells transduction was performed after $48 \mathrm{~h}$. Retrovirus was produced from $293 \mathrm{~T}$ cell lines. T cells were transduced with retrovirus supernatants in retronectin (Takara, Otsu, Japan)-coated plates. To enhance transduction efficiency, the plates were centrifuged with $3000 \mathrm{rpm}$ for $90 \mathrm{~min}$.

\section{In vitro Cytotoxic Assay}

CAR-T cells were co-cultured with $5 \times 10^{4}$ target cells at different effector/target $(\mathrm{E} / \mathrm{T})$ ratio in target cell medium in black-walled 96 well plates. After $18 \mathrm{~h}$, the cells were treated with D-luciferin (GoldBio, St. Louis, United States). Emitted light was detected by a luminescence plate reader (Thermo Varioskan Flash). Lysis percentage was calculated as [1(luminescence intensity of each sample)/(luminescence intensity of target cells alone)] $\times 100$.

\section{Flow Cytometry}

For cytokines and exhaustion markers analysis, CAR-T cells were stimulated with irradiated $8505 \mathrm{c}$-TSHR cells for $24 \mathrm{~h}$. The BD Cytometric Bead Array (CBA) kit were used to quantify the secretion level of IL-2, TNFa and IFN- $\gamma$ of the supernatant of CAR-T cells following manufacturer's instruction. For intracellular cytokines staining, the Golgi plug protein transport inhibitor Brefeldin A (eBioscience, San Diego, United States) was added into the cultured medium $4 \mathrm{~h}$ before detection. T cells were then fixed and permeabilized with Fixation and Permeabilization kit (BD Biosciences, San Jose, United States) according to manufacturer's instruction. The following antibodies were used: FITC mouse anti-human TSHR (Santa Cruz Biotechnology, Santa Cruz, United States), Human IL-2 Flex Set (BD Biosciences), Human TNF Flex Set (BD Biosciences), Human IFN- $\gamma$ Flex Set (BD Biosciences), BV421 rat anti-human IL-2 (BD Biosciences), PE-Cy7 mouse antihuman TNFa (BD Biosciences), PE-Cy7 anti-human CD279 (PD-1) (eBioscience), eFluor 450 anti-human CD223 (LAG-3) (eBioscience). Flow cytometry was performed on a CytoFLEX LX cytometer (Beckman Coulter, Brea, United States). A moflo Astrios EQ cell sorter (Beckman Coulter) was used for cell sorting. Data were analyzed with FlowJo software (FlowJo LLC, Ashland, United States).

\section{Animal Experiments}

Six- to 12 -week-old NOD/SCID/IL-2R $\gamma^{\text {null }}$ (NSG) mice were obtained from Jihui Shanghai and housed in the Animal Core Facility at Westlake University. All procedures followed the Institutional Animal Care and Use Committee (IACUC) guideline. For the in vivo anti-tumor assay, mice were intravenously injected with $1 \times 10^{6} \quad 8505 \mathrm{c}$-TSHR cells followed by $0.5 \times 10^{6} \mathrm{CAR}-\mathrm{T}$ cells the next day. To monitor tumor burden, mice were treated with $200 \mu \mathrm{L}$ of $15 \mathrm{mg} / \mathrm{ml}$ D-luciferin by intraperitoneal injection. Bioluminescence imaging was performed using the Optima small animal imaging system (Biospace Lab, Paris, France).

\section{Image Acquisition and Analysis}

Time-lapse imaging was performed with a Nikon Eclipse Ti inverted microscope. A Tokai Hit ST Series Stage Top Incubator was used to maintain a $5 \% \mathrm{CO}_{2}$ at $37^{\circ} \mathrm{C}$ for cells during imaging. The W-VIEW GEMINI imaging splitting optics (Hamamatsu Photonics, Hamamatsu, Japan) with a 438/29 nm excitation filter, a $474 / 40 \mathrm{~nm}$ emission filter, a $535 / 25 \mathrm{~nm}$ emission filter, an iXon Ultra 897 EMCCD camera (Andor Technology, Belfast, United Kingdom) was used to acquire the ECFP and FRET fluorescent signals simultaneously. CAR-Jurkat T cells co-expressing ZAP70 or ERK biosensor were generated by lentivirus transduction. To monitor ZAP70 or ERK signal dynamics upon antigen stimulation, CAR-Jurkat $\mathrm{T}$ cells were dropped on the glass-bottom dishes coated with K562-TSHR cells. From that time on, images were acquired at an interval of $30 \mathrm{~s}$ for $40 \mathrm{~min}$. The imaging data were analyzed by Fluocell software. The ECFP/FRET ratio of ZAP70 biosensor or the FRET/ ECFP ratio of ERK biosensor for each cell was normalized before comparison.

\section{Statistics}

Statistical analysis was performed using GraphPad Prism 8 software. An unpaired, two-tailed student's $t$-test were used to 


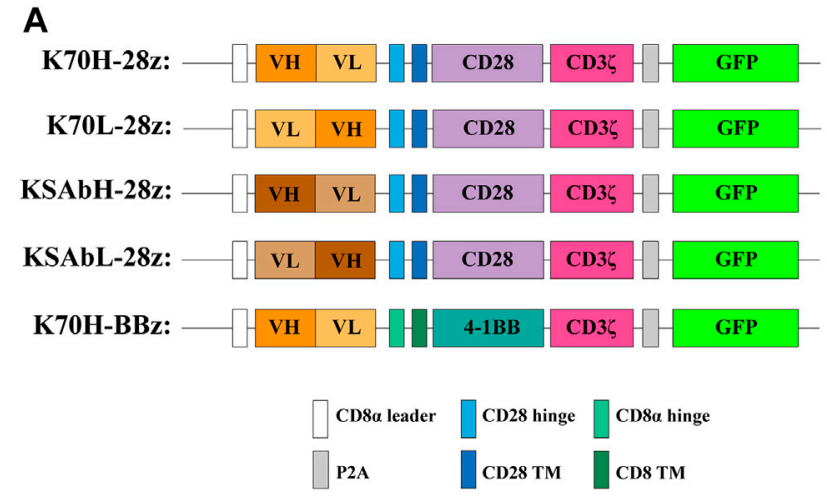

C

In Vitro killing assay (8505c-TSHR)

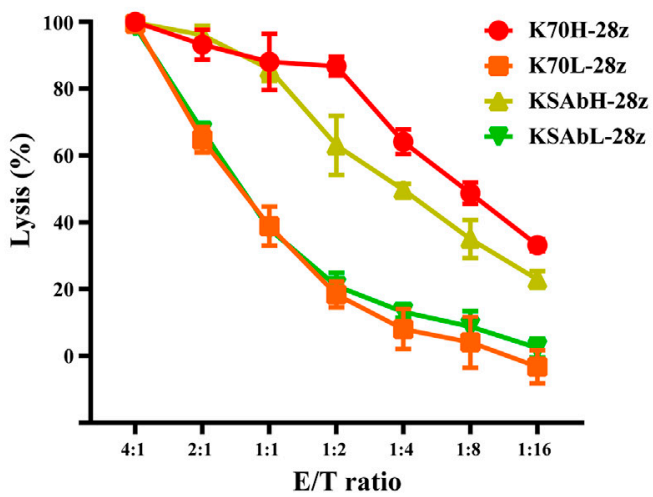

B
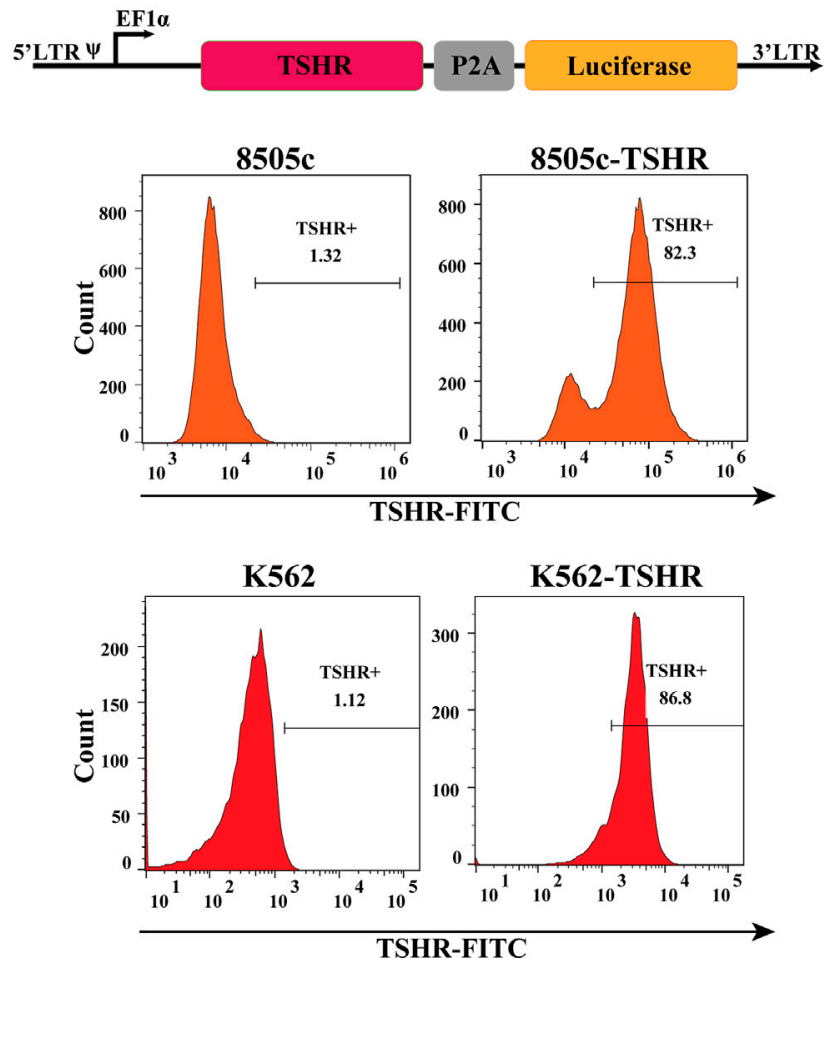

FIGURE 1 | Design of TSHR-specific CARs. (A) Construction of CARs with different scFvs and co-stimulatory domains. TM: transmembrane. (B) Generation of 8505c and K562 cell lines expressing TSHR antigen. (C) In vitro killing assay of primary CAR-T cells with different scFvs. Error bars: mean \pm SD.

determine the statistical differences between two groups. A Kaplan-Meier curve and the log-rank test were used to compare the survival differences between the groups in animal experiments. A $p$ value $<0.05$ was considered to be statically significant.

\section{RESULTS}

\section{Design of CAR Constructs Targeting TSHR}

Based on a second-generation CAR construct we investigated the feasibility of CAR-T cells in treating TSHR-positive thyroid cancers. In our design, the ectodomain of CARs was a single chain variable fragment $(\mathrm{scFv})$ that recognize TSHR, and the endodomain contained a CD28 or 4-1BB-derived costimulatory domains and $\mathrm{CD} 3 \zeta$-derived activation domain. To evaluate the expression level of CARs, a green fluorescence protein (GFP) was introduced downstream of CAR through a P2A self-cleavage peptide (Figure 1A). Although not strictly correlated, the expression of GFP could be used to estimate CAR expression level. To find the optimized scFvs, we constructed and compared scFvs from two antiTSHR monoclonal antibodies (K1-70 and KSAb) that have been previously reported. As the arrangement of variable heavy chain $(\mathrm{VH})$ and variable light chain (VL) fragment may largely affect the antigen recognition property, scFvs with different VH-VL arrangements were constructed for each antibody. Thus, four CD28-based CAR constructs with different scFvs (referred to $\mathrm{K} 70 \mathrm{H}-28 \mathrm{z}, \mathrm{K} 70 \mathrm{~L}-28 \mathrm{z}, \mathrm{KSAbH}-28 \mathrm{z}$ and KSAbL-28z) were generated (Figure 1A).

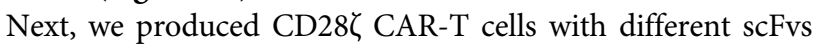
from primary $\mathrm{T}$ cells and compared their cytotoxic effect. Since most thyroid cell lines have lost their TSHR expression (Li et al., 2021), we established a TSHR-positive target cell line by transducing the full-length TSHR gene with a luciferase reporter into the thyroid cancer cell line 8505c (8505c-TSHR) (Figure 1B). Using 8505c-TSHR as the target cell, in vitro killing assay revealed the superior cytotoxic effect of K70H-28z CAR-T cells than its counterparts (Figure 1C). Therefore, the K1-70 derived VH-VL scFv was selected for subsequent studies.

\section{In vitro Cytotoxicity of TSHR CAR-T Cells With CD28 or 4-1BB Co-Stimulatory Domain} Nowadays, the most widely used CARs in clinic contain either CD28 or $4-1 \mathrm{BB}$ as co-stimulatory domains. Both of them have achieved impressive results in clinical studies. However, CAR-T cells bearing CD28 or 4-1BB intracellular domains could have different cytotoxic functions and in vivo persistence (Feucht and 
A
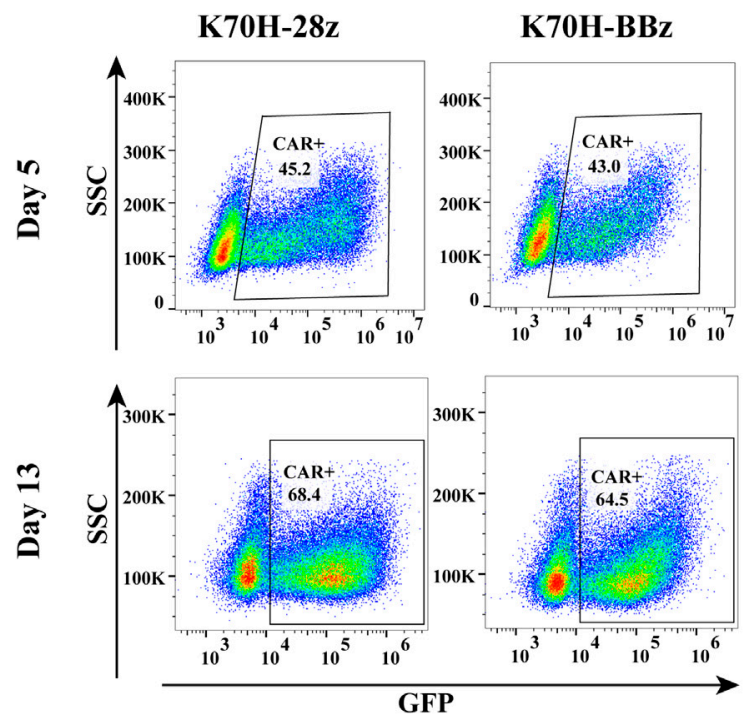

B
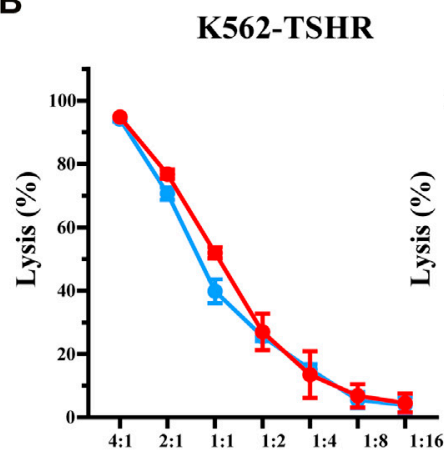

E/T ratio

C

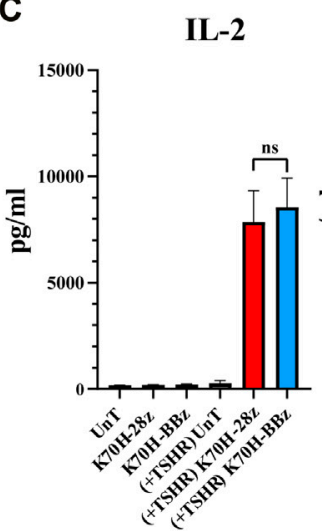

8505c-TSHR

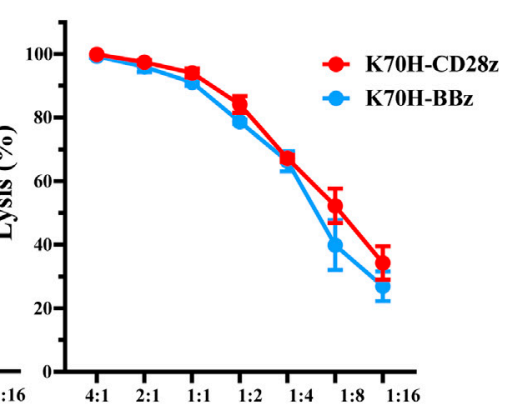

E/T ratio
D
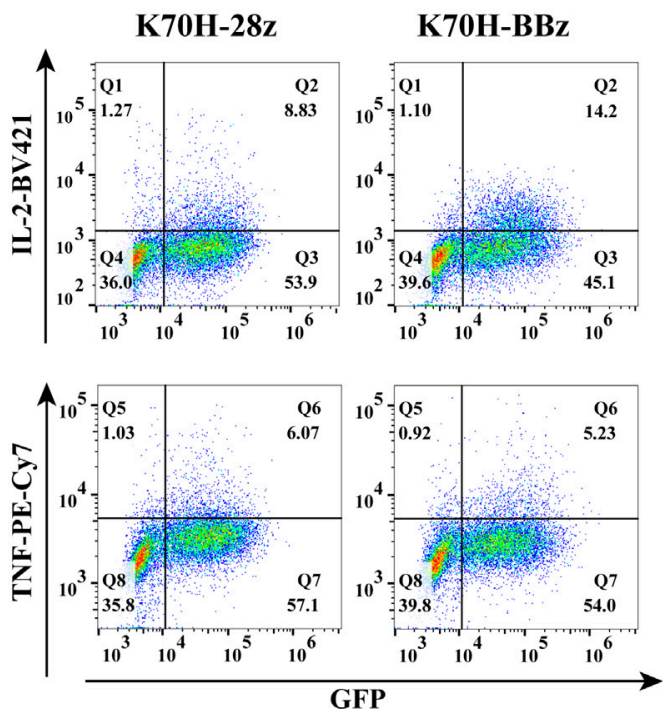

E

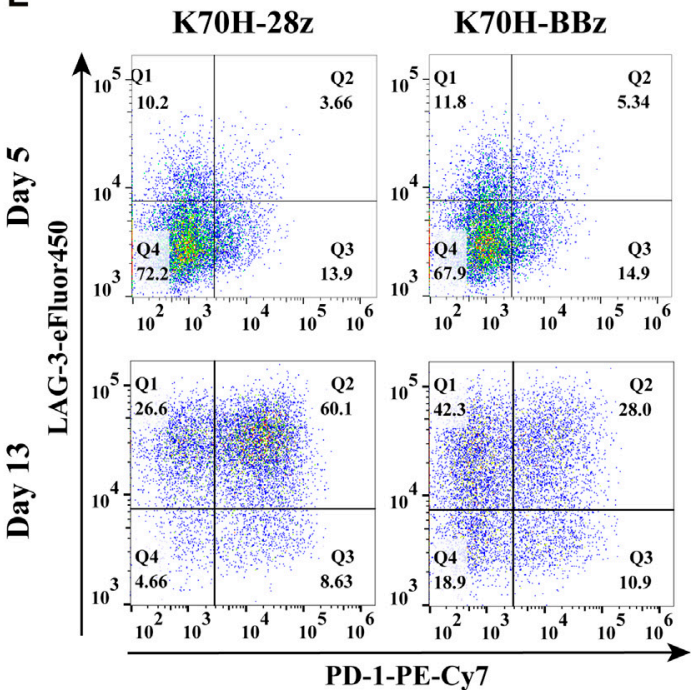

FIGURE 2 | In vitro characterization of CD28 and 4-1BB-based TSHR CAR-T cells. (A) CAR expression of primary T cells after retrovirus transduction. T cells were collected at day 5 and 13 after PBMC isolation and evaluated by FACS. CAR expression was indicated by GFP intensity of T cells. (B) In vitro killing assay of different CART cells against indicated target cells. (C-E) CAR-T cells were co-cultured with irradiated 8505c-TSHR cells for 24 h. The cytokine secretion of the supernatant (C), the intracellular cytokines expression (D) and the exhaustion genes expression (E) of CAR-T cells were evaluated by FACS. UnT: Untransduced T cells. Error bars: mean \pm SD.

Sadelain, 2020). To find out the optimal co-stimulatory domain for thyroid cancer treatment, we compared the in vitro antitumor functions of CD28 and 4-1BB-based TSHR CAR-T cells.
The CAR structures $(\mathrm{K} 70 \mathrm{H}-28 \mathrm{z} \& \mathrm{~K} 70 \mathrm{H}-\mathrm{BBz})$ have the same $\mathrm{scFv}$ but different hinge and transmembrane domains (Figure 1A). Produced by retrovirus transduction, both CAR- 
A

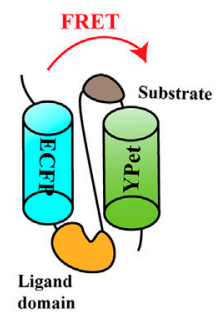

$\stackrel{\text { ZAP70 Kinase }}{\longrightarrow}$

$\overleftarrow{\text { Phosphatase }}$

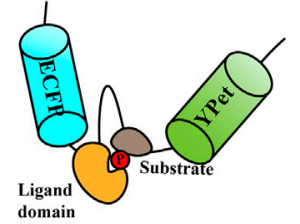

C Time (min) 0
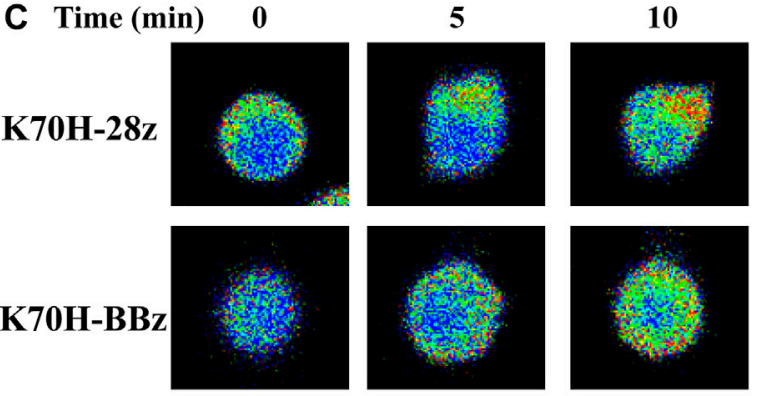

D

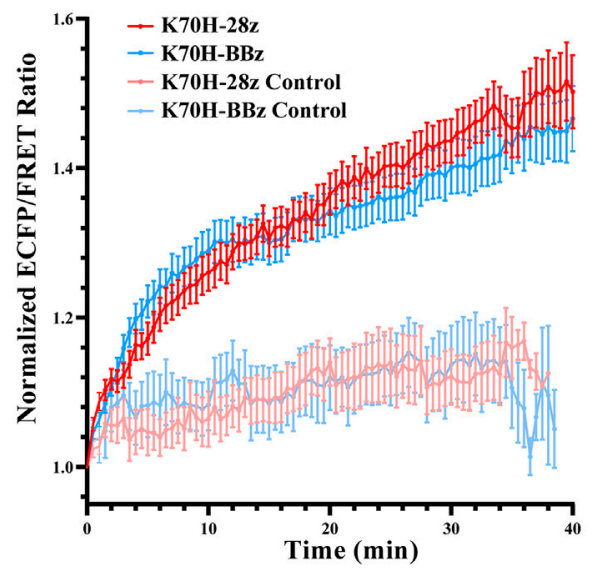

B 5, LTR $\Psi \stackrel{\text { EF10 }}{\Gamma}$
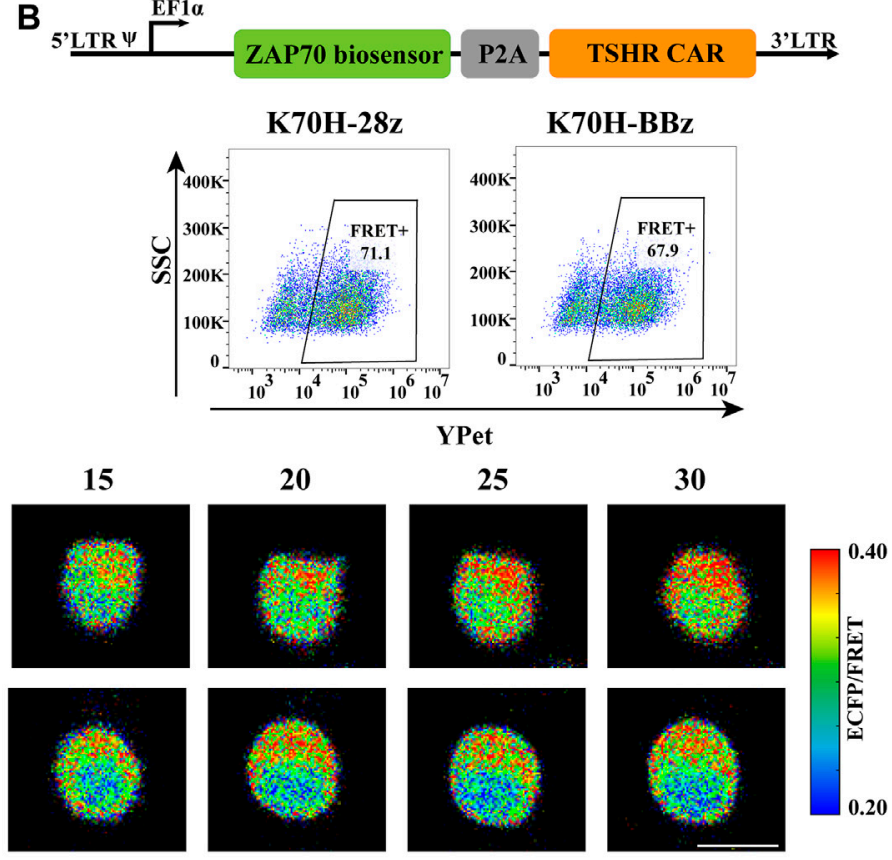

E
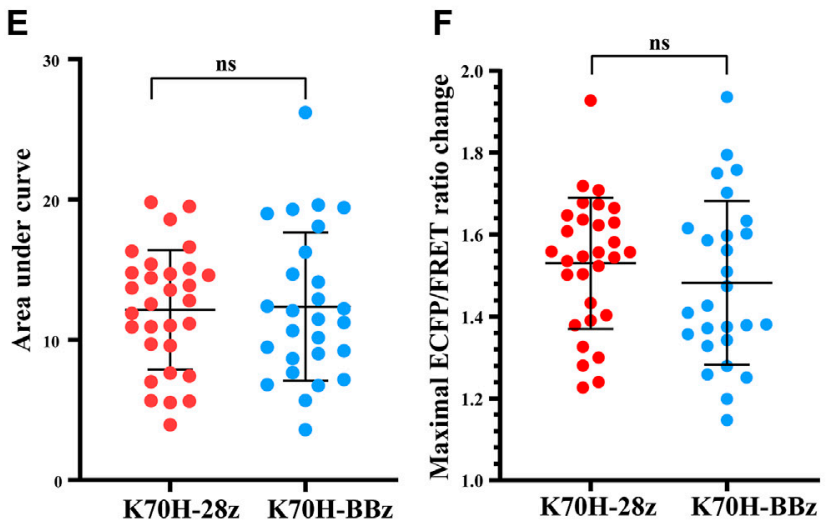

FIGURE 3 | Signaling dynamics of ZAP70 in CAR-T cells upon antigen stimulation. (A) Design of ZAP70 FRET biosensor. (B) Biosensor (CAR) expression of Jurkat T cells transduced with ZAP70 biosensor and TSHR CARs. (C) ZAP70 signal changes of CAR-Jurkat T cells over time after contacting with K562-TSHR cells. The activity of ZAP70 was visualized by ECFP/FRET ratio. Scale bar: $10 \mu \mathrm{m}$. (D) Time courses of ECFP/FRET ratio of ZAP70 biosensor in K70H-28z or K70H-BBz CAR-Jurkat T cells stimulated by K562-TSHR ( $n=30$ and 27 respectively) or K562 (Control, $n=10$ and 10 respectively). Error bars: mean \pm SEM. Accumulated signal intensity (area under curve) (E) and maximal ECFP/FRET ratio changes (F) of ZAP70 biosensor in CAR-Jurkat T cells. Error bars: mean \pm SD.

T cells showed similar transduction efficiency (Figure 2A). Using either K562-TSHR (Figure 1B) or 8505c-TSHR as target cells, their cytotoxicity was compared by in vitro killing assay. We found comparable cytotoxic effect between $\mathrm{K} 70 \mathrm{H}-28 \mathrm{z}$ and $\mathrm{K} 70 \mathrm{H}-$ $\mathrm{BBz}$ CAR-T cells (Figure 2B). We then analyzed the cytokines release of CAR-T cells by FACS. Co-culture with 8505c-TSHR cells promoted $\mathrm{K} 70 \mathrm{H}-28 \mathrm{z}$ and $\mathrm{K} 70 \mathrm{H}-\mathrm{BBz} \mathrm{CAR}-\mathrm{T}$ cells but not the untransduced $\mathrm{T}$ cells to secrete robust IL-2, TNFa and IFN $\gamma$ (Figure 2C). The $\mathrm{K} 70 \mathrm{H}-28 \mathrm{z}$ CAR-T cells secreted comparable levels of IL-2 and IFN $\gamma$, but higher levels of TNFa than K70H-
$\mathrm{BBz}$ CAR-T cells (Figure 2C). Intracellular cytokines staining further confirmed that antigen stimulation notably induced $\mathrm{GFP}^{+}$ CAR-T cells to secrete IL-2 and TNFa but not $\mathrm{GFP}^{-} \mathrm{T}$ cells (Figure 2D).

After a period of ex vivo culture, which is often required for CAR-T cell expansion before infusion, we observed an increased $\mathrm{GFP}^{+}$ratio of the total populations, representing an enrichment of the CAR-T cells. To evaluate the exhaustion levels, we detected PD-1 and LAG-3 expression of CAR-T cells after antigen stimulation (Figure 2E). Compared to CAR-T cells at day 5, 
A

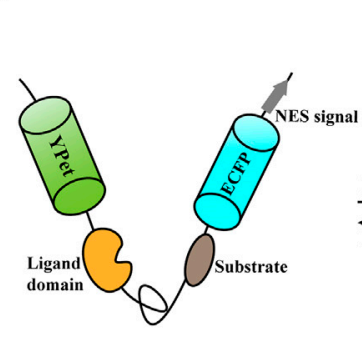

C Time (min) 0

K70H-28z

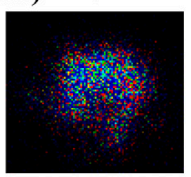

K70H-BBz
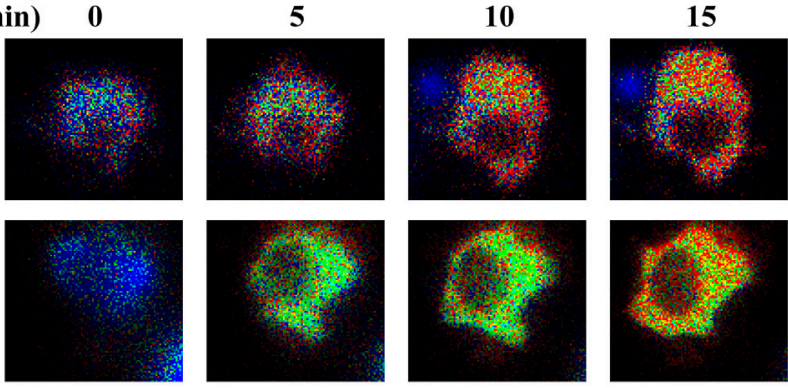

E

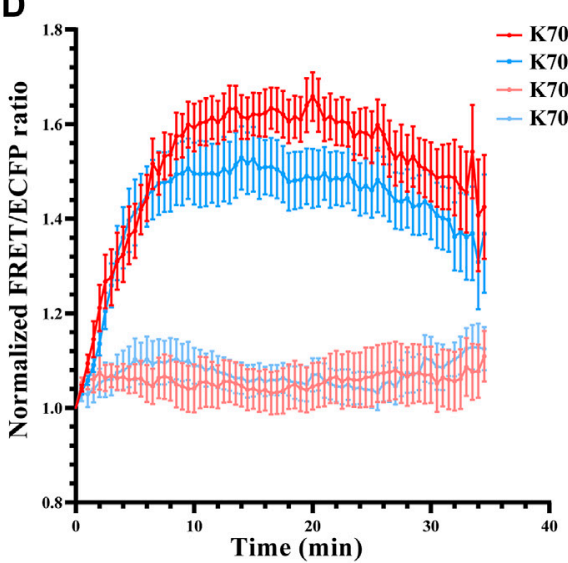

B 5'LTR $\psi \stackrel{\text { EF1 }}{\Gamma}$

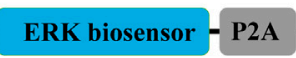

TSHR CAR

$\stackrel{\text { 3'LTR }}{\longrightarrow}$

K70H-28z

K70H-BBz
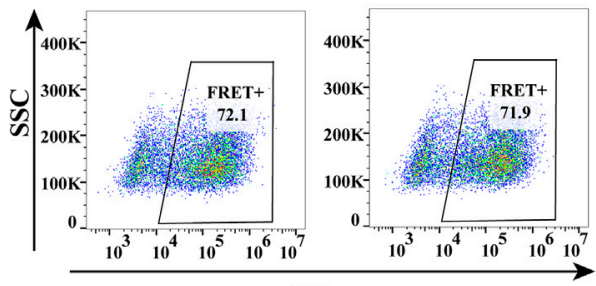

YPet

20
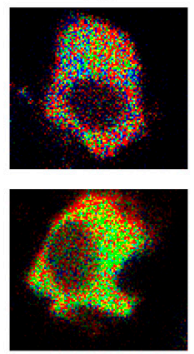

25
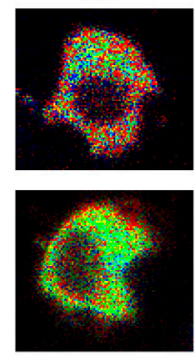

F

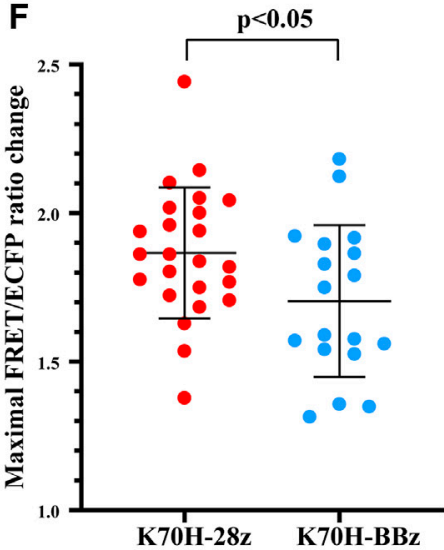

FIGURE 4 | Signaling dynamics of ERK in CAR-T cells upon antigen stimulation. (A) Design of ERK FRET biosensor. (B) Biosensor (CAR) expression of Jurkat T cells transduced with ERK biosensor and TSHR CARs. (C) ERK signal changes of CAR-Jurkat T cells over time after contacting with K562-TSHR cells. The activity of ERK was visualized by FRET/ECFP ratio. Scale bar: $10 \mu \mathrm{m}$. (D) Time courses of FRET/ECFP ratio of ERK biosensor in K7OH-28z or K7OH-BBz CAR-Jurkat T cells stimulated by K562-TSHR ( $n=24$ and 18 respectively) or K562 (Control, $n=14$ and 14 respectively). Error bars: mean \pm SEM. Accumulated signal intensity (area under curve) (E) and maximal FRET/ECFP ratio changes (F) of ERK biosensor in CAR-Jurkat T cells. Error bars: mean \pm SD.

CAR-T cells at day 13 showed increased expression of both exhaustion markers (Figure 2E). Moreover, $60.1 \%$ of the K70H-28z CAR-T cells showed double positive of PD-1 and LAG-3, much higher than that of the $\mathrm{K} 70 \mathrm{H}-\mathrm{BBz}$ group (Figure 2E).

\section{Similar ZAP70 Activation Downstream of CD28 or 4-1BB-Based TSHR CARs Revealed by FRET Imaging}

Although previous studies have characterized different signal activities triggered by CD28 or 4-1BB-based CARs (Philipson et al., 2020), their signaling dynamics in the early stage of T cell activation are still elusive. To figure out how co-stimulatory molecules affect CAR-induced signal transductions, we first focused on the membrane-proximal signaling events that were activated at the earliest time after antigen stimulation. ZAP70 has been considered as one of the most important kinases to transduce CAR signals into downstream signaling cascades (Wang et al., 2010; Gudipati et al., 2020). We have applied FRET-based ZAP70 biosensor to monitor the activity changes of ZAP70 during TSHR CAR-T cells activation. The ZAP70 biosensor was designed to include a FRET pair of ECFP and YPet fluorescence proteins, a ZAP70 kinase-specific substrate 
A

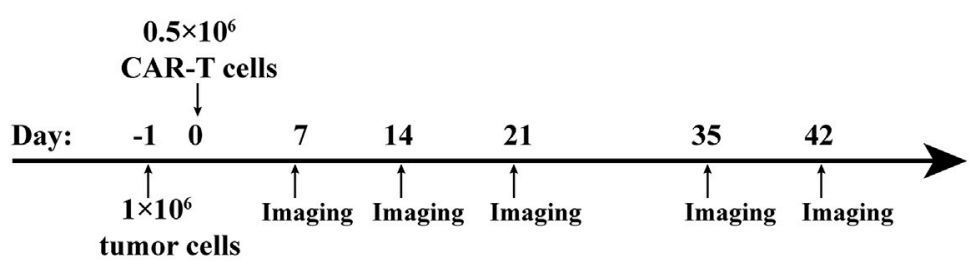

B

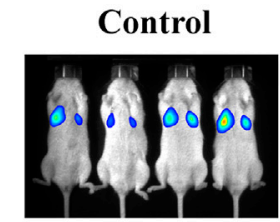

Day 7

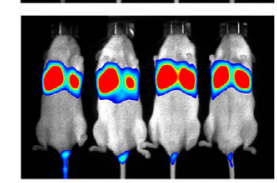

Day 14

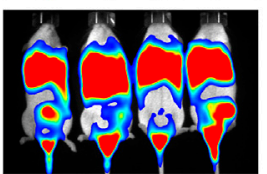

Day 21

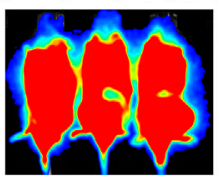

Day 35

Day 42
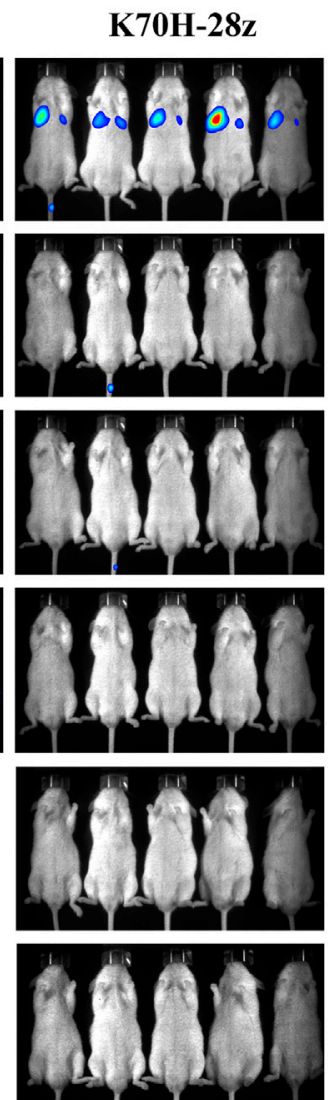

K70H-BBz
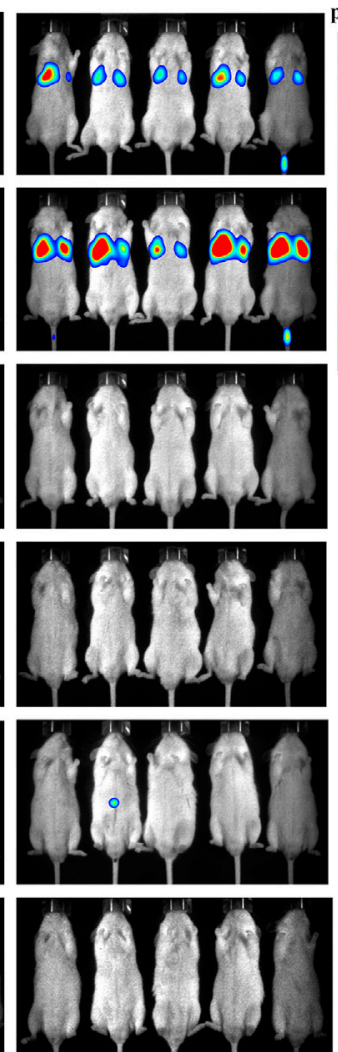

C

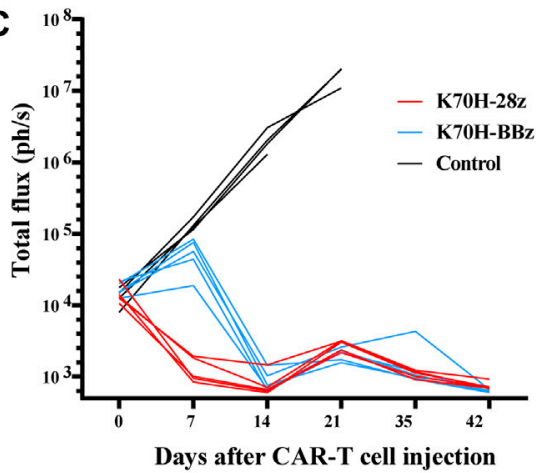

D

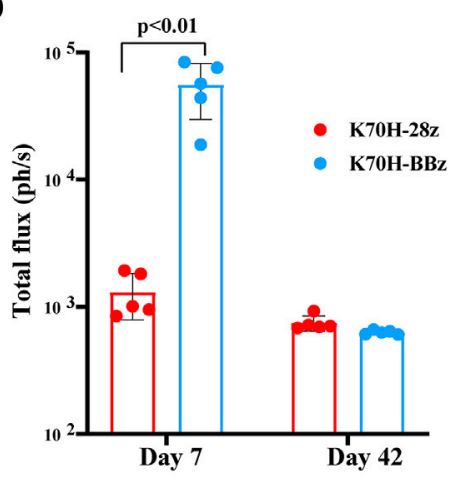

E

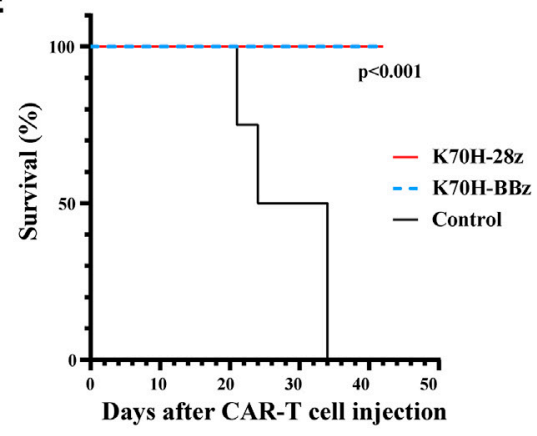

FIGURE 5 | In vivo anti-tumor functions of TSHR CAR-T cells. (A) Schematic timeline of the in vivo experimental design. NSG mice were intravenously injected with $1 \times 10^{6} 8505 \mathrm{c}-\mathrm{TSHR}$ cells. The next day, they were treated with $0.5 \times 10^{6} \mathrm{~K} 70 \mathrm{H}-28 \mathrm{z} \mathrm{CAR}-\mathrm{T}$ cells $(n=5), \mathrm{K} 70 \mathrm{H}-\mathrm{BBz} \mathrm{CAR}-\mathrm{T}$ cells $(n=5)$ or PBS $(n=4)$. (B) Bioluminescence imaging of mice at indicated days after treatment. (C) Quantification of tumor burden of mice. (D) Tumor burden of K7OH-28z CAR and K7OH-BBz CAR-T groups at day 7 and 42. Error bars: mean \pm SD. (E) Kaplan-Meier analysis of survival of mice from different groups.

domain, and a ligand domain. Phosphorylation of the substrate by ZAP70 would cause conformational changes of the biosensor, resulting in reduction of FRET efficiency between ECFP and YPet (Figure 3A) (Liu et al., 2021). Therefore, the relative activity of ZAP70 could be evaluated by detecting the fluorescence ratio of ECFP and FRET (YPet). To assess the ZAP70 activity upon antigen stimulation, the Jurkat $\mathrm{T}$ cell line co-expressing ZAP70 biosensor and CARs (CAR-Jurkat $\mathrm{T}$ ) were generated by lentivirus transduction. Both $\mathrm{K} 70 \mathrm{H}-28 \mathrm{z}$ and $\mathrm{K} 70 \mathrm{H}-\mathrm{BBz}$ CAR-Jurkat T cells showed similar expression of biosensor (CAR) (Figure 3B). By FRET imaging, we observed remarkable changes of the ZAP70 activity in CAR-Jurkat T cells upon encountering with the K562TSHR target cells (Figure 3C). The ZAP70 activity increased rapidly within $10 \mathrm{~min}$ after antigen stimulation. Thereafter, its signal continued to increase with slower speed (Figure 3D). We further quantified the accumulated signal intensity (area under curve) and maximal signal intensity (maximal ECFP/FRET ratio changes) of $\mathrm{K} 70 \mathrm{H}-28 \mathrm{z}$ and $\mathrm{K} 70 \mathrm{H}-\mathrm{BBz}$ CARs. Neither the accumulated signal intensity nor the maximal signal intensity of ZAP70 between $\mathrm{K} 70 \mathrm{H}-28 \mathrm{z}$ and $\mathrm{K} 70 \mathrm{H}-\mathrm{BBz}$ CARs showed significant difference (Figures 3E,F).

\section{Different ERK Activation Dynamics in TSHR CAR-T Cells Revealed by FRET Imaging}

To assess whether signals downstream of ZAP70 were affected by different co-stimulatory molecules, we investigated the signal dynamics of ERK during CAR-T cells activation by FRET- 
based biosensor. The ERK biosensor used a flexible linker to connect the substrate, ligand domain and FRET pairs. It also included a nucleus exporting signal (NES) to increase detection sensitivity of cytosolic signal. The biosensor would increase its FRET efficiency when phosphorylated by ERK (Figure 4A) (Komatsu et al., 2011). The CAR-Jurkat T cells expressing ERK biosensor and $\mathrm{K} 70 \mathrm{H}-28 \mathrm{z}$ or $\mathrm{K} 70 \mathrm{H}-\mathrm{BBz}$ CAR were generated by lentivirus transduction and showed similar expression of biosensor (CAR) between groups (Figure 4B). By FRET imaging we found that the ERK activity, as visualized by FRET/ECFP fluorescence ratio of CAR-Jurkat $\mathrm{T}$ cells increased upon K562-TSHR cells stimulation (Figure 4C). Statistical quantification suggested that the activity of ERK reached to a peak level in about $15 \mathrm{~min}$ and then underwent slow decrease (Figure 4D). The accumulated signal intensity of ERK in $\mathrm{K} 70 \mathrm{H}-28 \mathrm{z}$ CAR was slightly higher than that of $\mathrm{K} 70 \mathrm{H}-\mathrm{BBz} \mathrm{CAR}$ but without significant difference (Figure 4E). However, the maximal signal intensity of ERK in $\mathrm{K} 70 \mathrm{H}-28 \mathrm{z}$ CAR was significantly higher than that of $\mathrm{K} 70 \mathrm{H}-\mathrm{BBz}$ CAR $(p<0.05)$ (Figure 4F). Collectively, compared with K70H$\mathrm{BBz}$ CAR, K70H-28z CAR triggered similar ZAP70 signaling but significantly enhanced ERK signaling.

\section{Different Anti-Tumor Kinetics of CD28 and 4-1BB-Based CAR-T Cells in vivo}

Finally, we evaluated the anti-tumor functions of $\mathrm{K} 70 \mathrm{H}-28 \mathrm{z}$ and $\mathrm{K} 70 \mathrm{H}-\mathrm{BBz} \mathrm{CAR}-\mathrm{T}$ cells by in vivo studies (Figure 5A). To mimic the distant metastasis of DTCs, of which lung and bone are the major sites, we established a xenograft animal model based on previous studies (Zhang et al., 2014). The 8505c-TSHR cells were intravenously injected into the immunodeficient NSG mice. We found the tumors cells accumulated in the lung at early stage. Bone and other tissue metastasis happened after 14 days and caused animal death in the control group (Figures 5B,C,E). By contrast, mice treated with either $\mathrm{K} 70 \mathrm{H}-28 \mathrm{z}$ or $\mathrm{K} 70 \mathrm{H}-\mathrm{BBz} \mathrm{CAR}-$ T cells successfully controlled tumor growth and survived without obvious side-effect (Figures 5B,C,E). Nevertheless, K70H-28z CAR-T cells were found to eradicate nearly all tumor cells within 7 days, while $\mathrm{K} 70 \mathrm{H}-\mathrm{BBz}$ CAR-T cells lagged behind (Figures 5C,D). This indicated faster anti-tumor kinetics of $\mathrm{K} 70 \mathrm{H}-28 \mathrm{z}$ CAR-T cells than $\mathrm{K} 70 \mathrm{H}-\mathrm{BBz}$ CAR-T cells.

\section{DISCUSSION}

In this study, we developed a CAR-T therapy for TSHR-positive advanced thyroid cancers. The CAR constructs were designed to bear a monoclonal antibody-derived scFv targeting TSHR and a CD28 or 4-1BB co-stimulatory domain. Primary T cells equipped with the CD28 or 4-1BB-based CARs were demonstrated with potent anti-tumor efficacy both in vitro and in vivo. Moreover, we applied the FRET biosensors for ZAP70 and ERK to monitor the signaling dynamics of CAR-T cells in response to antigen stimulation. These results revealed different ERK activation properties of CD28 and 4-1BB-based CAR-T cells.
To recognize TSHR-positive tumor cells, we constructed scFvs from two monoclonal antibodies, K1-70 and KSAb. Both antibodies were derived from patients with Graves' disease. They have an estimated affinity of 25 and 22 pM for human TSHR, respectively (Sanders et al., 2010; Banga et al., 2013). The slightly higher affinity of K1-70 may contribute to the stronger cytotoxicity of $\mathrm{K} 70 \mathrm{H}-28 \mathrm{z}$ CAR-T cells than KSAbH-28z CAR-T cells. Despite the potent anti-tumor effect of $\mathrm{K} 70 \mathrm{H}$-derived CAR$\mathrm{T}$ cells, previous studies have suggested that the affinity of scFvs did not positively correlate with CAR-T cells functions (Ghorashian et al., 2019). The increased affinity of scFvs above threshold may not improve $\mathrm{T}$ cell activation but decrease selectivity. Fine-tuning of the scFv affinity may be required to maximize CAR-T cell functions as well as minimize possible ontarget/off-tumor toxicity (Di Roberto et al., 2020).

To transduce the antigen stimulation into intracellular signals responsible for $\mathrm{T}$ cell activation, the cytoplasmic domains of CARs have been incorporated with a $\mathrm{CD} 3 \zeta$ chain and a $\mathrm{CD} 28$ or 4-1BB derived co-stimulatory domain. Previous studies have suggested remarkable variations in functions of CAR-T cells equipped with various co-stimulatory domains. In CD19 CAR$\mathrm{T}$ cells studies, $28 \mathrm{z}$ CAR- $\mathrm{T}$ cells exhibited higher effector functions with less persistence than $\mathrm{BBz}$ CAR-T cells (Shah and Fry, 2019; Ying et al., 2019). This functional discrepancy has been associated with different metabolic features. 28z CAR-T cells have shown enhanced aerobic glycolysis, while BBz CAR-T cells have exhibited greater mitochondrial oxidative phosphorylation (Kawalekar et al., 2016). Interestingly, phosphoproteomic analysis have indicated that 28z CAR and $\mathrm{BBz}$ CAR induce $\mathrm{T}$ cells activation through common signaling intermediates but with different kinetics and intensities (Salter et al., 2018).

We detected the ZAP70 and ERK signal activities in CARJurkat $\mathrm{T}$ cells after antigen stimulation by FRET biosensors. Compared with methods like Western blot and mass spectrum, FRET biosensors could detect kinase activity in single live cells with high spatiotemporal resolution. This enables us to evaluate early signaling events of $\mathrm{T}$ cell activation. We observed the interaction with TSHR-positive tumor cells induced rapid increase of ZAP70 and ERK activities within minutes, indicating antigen-dependent activation of the CAR-Jurkat T cells. Different from previous studies, we did not observe significant difference of ZAP70 signal activities in $\mathrm{K} 70 \mathrm{H}-28 \mathrm{z}$ and $\mathrm{K} 70 \mathrm{H}-\mathrm{BBz}$ CAR-T cells after activation (Salter et al., 2018; Philipson et al., 2020). One main reason responsible for these results may be the different detection methods. In previous studies, ZAP70 activity was evaluated by the phosphorylation level of some key phosphorylated sites like Y319. However, the phosphorylation state of the specific site did not precisely reflect the activity of kinase. In contrast, the FRET ratio change of FRET biosensors was determined by the phosphorylation of ZAP70-specific substrate, which could more accurately reflect the activation state of ZAP70. In addition, the previous comparison was done between CD19targeting $28 \mathrm{z}$ and $\mathrm{BBz}$ CAR-T cells while in this study, TSHRtargeting CAR-T cells have different scFvs and CAR targets. 
Nevertheless, ZAP70 activation mainly depends on CD3 $\zeta$ instead of co-stimulatory molecules, which matched our results.

Consistent with previous studies, we observed an enhanced signal activity of ERK in $28 \mathrm{z}$ CAR-T cells when compared to $\mathrm{BBz}$ CAR-T cells (Salter et al., 2018). CD28 and 4-1BB as costimulatory molecules are thought to amplify ERK signaling with distinct mechanisms. CD28 may enhance ERK signaling by promoting the recruitment of Vav-1 to the plasma membrane, which is required for Rac-1 activation (Michel et al., 2000). 4-1BB may induce ERK activation via its downstream receptor TRAF2 to regulate Tpl2 activation (Xie et al., 2021). In CAR-T cells it is still elusive how CARs with different co-stimulatory molecules mediate the activation of ERK signal. Diverse signaling output derived from CD28 or 4-1BB may contribute to the amplification of ERK pathway. As observed in our studies, the different ERK activities of $\mathrm{K} 70 \mathrm{H}-28 \mathrm{z}$ and $\mathrm{K} 70 \mathrm{H}-\mathrm{BBz}$ CAR-T cells may induce large distinction of downstream signals and contribute to different anti-tumor functions. Notably, we have observed that the ERK signal reached a peak value at about 15 min after antigen engagement, at which time ZAP70 signal also showed a short plateau. Thereafter, the increased ZAP70 activities did not promote further enhancement of ERK signals. The correlation of ZAP70 and ERK signals requires further investigation. In addition, single cell FRET imaging allowed us to observe heterogeneous activities of ZAP70 and ERK in individual CAR-T cells after antigen stimulation. This cell-to-cell heterogeneity obscured by traditional methods may be caused by distinct expression levels of CARs and related signaling molecules or different contacting strength with target cells. More importantly, this heterogeneity may lead to the diverse $\mathrm{T}$ cell differentiation and fates. However, with limited number of cells collected by FRET imaging, it is challenging to find possible links and correlations.

Although with comparable cytotoxic effects in vitro, we have shown that $\mathrm{K} 70 \mathrm{H}-28 \mathrm{z}$ CAR-T cells had higher expression of the exhaustion genes than $\mathrm{K} 70 \mathrm{H}-\mathrm{BBz}$ CAR-T cells. This may suggest the shorter persistence of $\mathrm{K} 70 \mathrm{H}-28 \mathrm{z}$ CAR-T cells. Meanwhile, the increased PD-1 and LAG-3 expression could be an indicator of $\mathrm{T}$ cell activation and the result of rapid effector functions of K70H-28z CAR-T cells (Wherry and Kurachi, 2015). Nevertheless, our in vivo experiments have observed potent anti-tumor functions of both designed CAR-T cells. Moreover, compared to $\mathrm{K} 70 \mathrm{H}-\mathrm{BBz}$ CAR-T cells, $\mathrm{K} 70 \mathrm{H}-28 \mathrm{z}$ CAR-T cells have shown rapid elimination of tumor cells, which suggested higher effector functions. It should be noticed that although longterm persistence of CAR-T cells in vivo is essential to avoid tumor relapse, it may also enhance the risk of off-tumor toxicity (Kawalekar et al., 2016). In the context of thyroid cancer treatment, it requires further studies to balance the effector functions and long-term persistence of CAR-T cells.

Finally, our studies have shown the efficacy of TSHR CAR-T cells in the treatment of TSHR-positive thyroid cancers. We also showed that CD28 and 4-1BB-based CAR-T cells exhibited comparable anti-tumor functions with different kinetics. Live cell imaging using FRET biosensors revealed the dynamics of ZAP70 and ERK activities in both CAR-T cells during activation. These results promoted our understanding of CAR-T cells signaling and indicated potential applications of FRET biosensors in optimizing CAR designs. CAR-T cells therapy may overcome current obstacles for thyroid cancer treatment in the near future.

\section{DATA AVAILABILITY STATEMENT}

All data obtained and/or analyzed during the current study are available from the corresponding authors on reasonable request.

\section{ETHICS STATEMENT}

The studies involving human participants were reviewed and approved by Ethics Committee of Zhejiang University School of Medicine. The patients/participants provided their written informed consent to participate in this study. The animal study was reviewed and approved by Institutional Animal Care and Use Committee of Westlake University.

\section{AUTHOR CONTRIBUTIONS}

JZ, XM and JS designed the study. JZ, JC and XM performed the experiments. $\mathrm{YH}, \mathrm{XG}$ and $\mathrm{CZ}$ provided experimental support and discussion. JZ, XM and JS wrote the manuscript. All authors read and approved the final manuscript.

\section{FUNDING}

This work was funded by the National Key R\&D Program of China 2021YFA0909900 (JS), National Natural Science Foundation of China grants 31971324 (JS), 81702650 (JZ); 81973993 (XG) and 31971125 (CZ), Natural Science Foundation of Hubei Province 2020CFB839 (JZ), Foundation of the Scientific and Technological Bureau of Wuhan 2020020601012327 (JZ) and by Zhejiang Provincial Natural Science Foundation grant LR20H160003 (JS).

\section{ACKNOWLEDGMENTS}

We thank the support of Zhejiang Provincial Key Laboratory of Immunity and Inflammatory diseases. 


\section{REFERENCES}

Banga, J.-P. S., Gilbert, J. A., Dunn-Walters, D., and Padoa, C. (2013). “Agonist Antibodies against TSHR," (Washington, DC: U.S. Patent and Trademark Office). U.S. Patent No 20100266493A1.

Bhoj, V. G., Li, L., Parvathaneni, K., Zhang, Z., Kacir, S., Arhontoulis, D., et al. (2021). Adoptive T Cell Immunotherapy for Medullary Thyroid Carcinoma Targeting GDNF Family Receptor Alpha 4. Mol. Ther. - Oncolytics 20, 387-398. doi:10.1016/j.omto.2021.01.012

Davies, T., Marians, R., and Latif, R. (2002). The TSH Receptor Reveals Itself. J. Clin. Invest. 110 (2), 161-164. doi:10.1172/jci0216234

Di Roberto, R. B., Castellanos-Rueda, R., Frey, S., Egli, D., Vazquez-Lombardi, R., Kapetanovic, E., et al. (2020). A Functional Screening Strategy for Engineering Chimeric Antigen Receptors with Reduced On-Target, Off-Tumor Activation. Mol. Ther. 28 (12), 2564-2576. doi:10.1016/j.ymthe.2020.08.003

Feucht, J., and Sadelain, M. (2020). Function and Evolution of the Prototypic

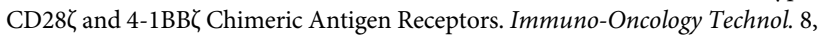
2-11. doi:10.1016/j.iotech.2020.09.001

Ghorashian, S., Kramer, A. M., Onuoha, S., Wright, G., Bartram, J., Richardson, R., et al. (2019). Enhanced CAR T Cell Expansion and Prolonged Persistence in Pediatric Patients with ALL Treated with a Low-Affinity CD19 CAR. Nat. Med. 25 (9), 1408-1414. doi:10.1038/s41591-019-0549-5

Gudipati, V., Rydzek, J., Doel-Perez, I., Gonçalves, V. D. R., Scharf, L., Königsberger, S., et al. (2020). Inefficient CAR-Proximal Signaling Blunts Antigen Sensitivity. Nat. Immunol. 21 (8), 848-856. doi:10.1038/s41590-0200719-0

Kawalekar, O. U., O’Connor, R. S., Fraietta, J. A., Guo, L., McGettigan, S. E., Posey, A. D., Jr., et al. (2016). Distinct Signaling of Coreceptors Regulates Specific Metabolism Pathways and Impacts Memory Development in CAR T Cells. Immunity 44 (2), 380-390. doi:10.1016/j.immuni.2016.01.021

Komatsu, N., Aoki, K., Yamada, M., Yukinaga, H., Fujita, Y., Kamioka, Y., et al. (2011). Development of an Optimized Backbone of FRET Biosensors for Kinases and GTPases. MBoC 22 (23), 4647-4656. doi:10.1091/mbc.E11-01-0072

Kosti, P., Maher, J., and Arnold, J. N. (2018). Perspectives on Chimeric Antigen Receptor T-Cell Immunotherapy for Solid Tumors. Front. Immunol. 9, 1104. doi:10.3389/fimmu.2018.01104

Laha, D., Nilubol, N., and Boufraqech, M. (2020). New Therapies for Advanced Thyroid Cancer. Front. Endocrinol. 11, 82. doi:10.3389/fendo.2020.00082

Li, H., Zhou, X., Wang, G., Hua, D., Li, S., Xu, T., et al. (2021). CAR-T Cells Targeting TSHR Demonstrate Safety and Potent Preclinical Activity against Differentiated Thyroid Cancer. J. Clin. Endocrinol. Metab. 9, dgab819. doi:10. 1210/clinem/dgab819

Li, K., Xiang, X., Sun, J., He, H.-T., Wu, J., Wang, Y., et al. (2016). Imaging Spatiotemporal Activities of ZAP-70 in Live T Cells Using a FRET-Based Biosensor. Ann. Biomed. Eng. 44 (12), 3510-3521. doi:10.1007/s10439-016-1683-6

Liu, L., Limsakul, P., Meng, X., Huang, Y., Harrison, R. E. S., Huang, T.-S., et al. (2021). Integration of FRET and Sequencing to Engineer Kinase Biosensors from Mammalian Cell Libraries. Nat. Commun. 12 (1), 5031. doi:10.1038/ s41467-021-25323-x

Michel, F., Mangino, G., Attal-Bonnefoy, G., Tuosto, L., Alcover, A., Roumier, A., et al. (2000). CD28 Utilizes Vav-1 to Enhance TCR-Proximal Signaling and NFAT Activation. J. Immunol. 165 (7), 3820-3829. doi:10.4049/jimmunol.165.7. 3820

Min, I. M., Shevlin, E., Vedvyas, Y., Zaman, M., Wyrwas, B., Scognamiglio, T., et al. (2017). CAR T Therapy Targeting ICAM-1 Eliminates Advanced Human Thyroid Tumors. Clin. Cancer Res. 23 (24), 7569-7583. doi:10.1158/10780432.CCR-17-2008

Newick, K., O’Brien, S., Moon, E., and Albelda, S. M. (2017). CAR T Cell Therapy for Solid Tumors. Annu. Rev. Med. 68, 139-152. doi:10.1146/annurev-med062315-120245

Philipson, B. I., O’Connor, R. S., May, M. J., June, C. H., Albelda, S. M., and Milone, M. C. (2020). 4-1BB Costimulation Promotes CAR T Cell Survival through
Noncanonical NF-Kb Signaling. Sci. Signal. 13 (625), eaay8248. doi:10.1126/ scisignal.aay 8248

Rowe, C. W., Paul, J. W., Gedye, C., Tolosa, J. M., Bendinelli, C., McGrath, S., et al. (2017). Targeting the TSH Receptor in Thyroid Cancer. Endocr. Relat. Cancer 24 (6), R191-R202. doi:10.1530/ERC-17-0010

Salter, A. I., Ivey, R. G., Kennedy, J. J., Voillet, V., Rajan, A., Alderman, E. J., et al. (2018). Phosphoproteomic Analysis of Chimeric Antigen Receptor Signaling Reveals Kinetic and Quantitative Differences that Affect Cell Function. Sci. Signal. 11 (544), eaat6753. doi:10.1126/scisignal.aat6753

Sanders, J., Núñez Miguel, R., Furmaniak, J., and Rees Smith, B. (2010). TSH Receptor Monoclonal Antibodies with Agonist, Antagonist, and Inverse Agonist Activities. Methods Enzymol. 485, 393-420. doi:10.1016/b978-0-12381296-4.00022-1

Shah, N. N., and Fry, T. J. (2019). Mechanisms of Resistance to CAR T Cell Therapy. Nat. Rev. Clin. Oncol. 16 (6), 372-385. doi:10.1038/s41571-0190184-6

Sung, H., Ferlay, J., Siegel, R. L., Laversanne, M., Soerjomataram, I., Jemal, A., et al. (2021). Global Cancer Statistics 2020: GLOBOCAN Estimates of Incidence and Mortality Worldwide for 36 Cancers in 185 Countries. CA A. Cancer J. Clin. 71 (3), 209-249. doi:10.3322/caac. 21660

van der Stegen, S. J. C., Hamieh, M., and Sadelain, M. (2015). The Pharmacology of Second-Generation Chimeric Antigen Receptors. Nat. Rev. Drug Discov. 14 (7), 499-509. doi:10.1038/nrd4597

Wan, R., Wu, J., Ouyang, M., Lei, L., Wei, J., Peng, Q., et al. (2019). Biophysical Basis Underlying Dynamic Lck Activation Visualized by ZapLck FRET Biosensor. Sci. Adv. 5 (6), eaau2001. doi:10.1126/sciadv.aau2001

Wang, H., Kadlecek, T. A., Au-Yeung, B. B., Goodfellow, H. E. S., Hsu, L. Y., Freedman, T. S., et al. (2010). ZAP-70: an Essential Kinase in T-Cell Signaling. Cold Spring Harbor Perspect. Biol. 2 (5), a002279. doi:10.1101/cshperspect.a002279

Wherry, E. J., and Kurachi, M. (2015). Molecular and Cellular Insights into T Cell Exhaustion. Nat. Rev. Immunol. 15 (8), 486-499. doi:10.1038/nri3862

Xiang, X., Sun, J., Wu, J., He, H.-T., Wang, Y., and Zhu, C. (2011). A FRET-Based Biosensor for Imaging SYK Activities in Living Cells. Cel. Mol. Bioeng. 4 (4), 670-677. doi:10.1007/s12195-011-0211-X

Xie, X., Zhu, L., Jie, Z., Li, Y., Gu, M., Zhou, X., et al. (2021). TRAF2 Regulates T Cell Immunity by Maintaining a Tpl2-ERK Survival Signaling axis in Effector and Memory CD8 T Cells. Cell Mol Immunol 18 (9), 2262-2274. doi:10.1038/ s41423-020-00583-7

Ying, Z., He, T., Wang, X., Zheng, W., Lin, N., Tu, M., et al. (2019). Parallel Comparison of 4-1BB or CD28 Co-stimulated CD19-Targeted CAR-T Cells for B Cell Non-hodgkin's Lymphoma. Mol. Ther. - Oncolytics 15, 60-68. doi:10. 1016/j.omto.2019.08.002

Zhang, L., Gaskins, K., Yu, Z., Xiong, Y., Merino, M. J., and Kebebew, E. (2014). An In Vivo Mouse Model of Metastatic Human Thyroid Cancer. Thyroid 24 (4), 695-704. doi:10.1089/thy.2013.0149

Conflict of Interest: The authors declare that the research was conducted in the absence of any commercial or financial relationships that could be construed as a potential conflict of interest.

Publisher's Note: All claims expressed in this article are solely those of the authors and do not necessarily represent those of their affiliated organizations, or those of the publisher, the editors, and the reviewers. Any product that may be evaluated in this article, or claim that may be made by its manufacturer, is not guaranteed or endorsed by the publisher.

Copyright (c) 2022 Zhou, Chen, Huang, Gao, Zhou, Meng and Sun. This is an openaccess article distributed under the terms of the Creative Commons Attribution License (CC BY). The use, distribution or reproduction in other forums is permitted, provided the original author(s) and the copyright owner(s) are credited and that the original publication in this journal is cited, in accordance with accepted academic practice. No use, distribution or reproduction is permitted which does not comply with these terms. 